Original Research Paper

\title{
A New Proposed Statistically-Derived Compromise Cut-off CD4 Count Value for HIV Patients to Start using ARVs
}

\author{
Maria Mokgadi Lekganyane and Solly Matshonisa Seeletse \\ Department of Statistics and Operations Research, Sefako Makgatho Health Sciences University, \\ PO Box 107, MEDUNSA, 0204, Gauteng Province, South Africa
}

\author{
Article history \\ Received: 08-01-2016 \\ Revised: 27-06-2016 \\ Accepted: 28-06-2016 \\ Corresponding Author: \\ Maria Mokgadi Lekganyane \\ Department of Statistics and \\ Operations Research, Sefako \\ Makgatho Health Sciences \\ University, PO Box 107, \\ MEDUNSA, 0204, Gauteng \\ Province, South Africa \\ Email:maria.lekganyane@smu.ac.za
}

\begin{abstract}
The study investigated the relationship between statistical outliers and the inconsistent values of the CD4 count recommended for starting the Antiretroviral Therapy (ART) by HIV-positive patients. Low CD4 counts imply a low immune system. It could be due to AIDS existence or closeness to death. An effective treatment to curb HIV impact is ART, which is recommended for low CD4 counts. However, countries differ in the values used. Developed nations recommend start of ART when the CD4 is still high in order to curb its development. Poor countries use very low CD4 counts. Some countries keep changing the CD4 value for this purpose. The problem is that when CD4 counts are too low, HIV may already be too advanced, making it difficult to save the patient from progression to full AIDS. There should be CD4 values derived using scientific methods to assist in standardizing the CD4 count value for ART commencement. Using a retrospective single-site cohort study design, the study analyses the CD4 counts using robust statistical methods together with conventional statistical methods to study outliers and then derive a compromise CD4 count value that could serve as the standard cut-off starting point for using ART. The CD4 count confidence limits showed outliers to be above 300 cells $/ \mathrm{mm}^{3}$. The lower bound of 0 cannot happen to any living person. The 300 upper bound is a value within the manageable outliers that has not reached critical HIV state. However, this value is near risky CD4 count values. The CD4 count of 300 cells $/ \mathrm{mm}^{3}$ indicates deteriorating HIV. If it is set as the starting point of taking ARVs, the patients involved can be saved from reaching painful states of lower CD4 counts.HIV patients' immune systems at this level can still be boosted without them showing physical weakness from eye inspection.
\end{abstract}

Keywords: ART, CD4, HIV, Outlier

\section{Introduction}

Curbing HIV/AIDS is undoubtedly desirable. ART was suggested for every HIV positive patient (Lichterfeld and Rosenberg, 2011). CD4 elicits ART use because low values signal HIV spread and immune system harm. ART are useful for HIV positive patients, especially for CD4 below 350 cells $/ \mathrm{mm}^{3}$. Poor countries recommend starting ART at 200 cells $/ \mathrm{mm}^{3}$. These values have also changed many times. Studying CD4 outliers is vital to manage HIV. Large outliers indicate HIV severity. Statistical modelling can help to detect severities in HIV, while robust methods help to understand the bulk of the data such that outliers do not distort meanings (Andersen, 2008). Robust statistical methods are specialised methods that can resist influences of outliers (Tofallis, 2008).

\section{CD4 Outliers}

An outlier is an observation lying very far from other values in a random sample, either being unreasonably low or high (Alqallaf et al., 2009). CD4 outliers are particularly important in identifying severe HIV status. Robust methods enable understanding the data even when outliers influence analyses. Outliers are not always clearly visible. When using traditional statistical methods, outliers may be masked and the estimates of residuals may be inflated (Maronna et al., 2006). In CD4 counts, outliers can distort actualities and severities may be obscured. Hence, the use of robust methods maintains 
meanings from bulks of data while identifying anomalies that may be revealing severities.

\section{Robust Statistical Methods}

Some efficient robust statistical methods developed along common traditional methods are: Least Absolute Deviation (LAD), Least Trimmed Squares (LTS), Sestimation and M-estimators (Strutz, 2010). Upgrading robust methods includes MM-estimation which combines the robustness of S-estimation with the efficiency of Mestimation. Robust methods can assist in outlier identification, which is sometimes difficult and ensuring that outliers do not distort the results (Dawson, 2011).

\section{Outlier Detection}

Let $Q_{1}$ and $Q_{3}$ be the first and third quartiles of a data set and $k$ a fence constant normally chosen to be either 1.5 or 3. According to Dovoedo (2011), Tukey's boxplots boundaries' method defines outliers as observations outside the interval with lower and upper boundaries:

$$
\begin{aligned}
& L=Q_{1}-k\left(Q_{3}-Q_{1}\right) \\
& U=Q_{3}+k\left(Q_{3}-Q_{1}\right)
\end{aligned}
$$

\section{HIV Treatment and Health}

The CD4 count of a healthy person ranges from 500 to 1,200 cells $/ \mathrm{mm}^{3}$. Below these values for HIV positive patients are signs for requiring ARVs. The U.S. Department of Health and Human Services (HHS) provides guidelines on using ARVs and other HIV medicines to treat HIV infection (Sieleunou and Souleymanou, 2009). These guidelines recommend that all HIV positive people should take ART and emphasise that everyone with a CD4 count below 350 cells $/ \mathrm{mm}^{3}$ should use ARVs. If an HIV-infected person's CD4 count drops rapidly or is below 200 cells $/ \mathrm{mm}^{3}$, starting ARV is imminent (Lichterfeld and Rosenberg, 2011). A CD4 increase is a sign of immune system recovery.

\section{Statistical Techniques}

\section{Robust Statistical Methods}

\section{Least Absolute Deviation}

Least Absolute Deviation (LAD) is a robust statistical optimization procedure comparable to the general Ordinary Least Squares (OLS) technique that approximates a dataset. To design the LAD problem, Wilcox (2011) proposes a data set of points $\left(x_{i}, y_{i}\right)$ with $i$ $=1,2, \ldots, n$ in which the problem is to find a function $f$ of a specific form containing parameters to be determined such that $y_{i} \approx f\left(x_{i}\right)$. The approach is to search for estimated values of the unknown parameters that minimise the sum of the absolute values of the residuals:

$$
S=\sum_{i=1}^{n}\left|y_{i}-f\left(x_{i}\right)\right|
$$

\section{M-Estimator}

M-estimators are a robust comprehensive class of estimators obtained as the minima of sums of data functions (van de Geer, 2000). Considering a family of probability density functions $f$ parameterized by $\theta$, a MLE of $\theta$ is calculated for each data set by maximising the likelihood function over the parameter space $\{\theta\}$. When the observations are independent and identically distributed, a MLE $\hat{\theta}$ satisfies:

$$
\hat{\theta}=\arg \max _{\theta}\left(\coprod_{i=1}^{n} f\left(x_{i}, \theta\right)\right)
$$

\section{Least Trimmed Squares}

Least Trimmed Squares (LTS) is a robust statistical method that fits a function to a data set $(\mathrm{Li}$, 2005). In OLS, the estimated parameter values $\beta$ are those values that minimise the objective function $S(\beta)$ of squared residuals:

$$
S=\sum_{i=1}^{n} r_{i}(\beta)^{2}
$$

For a LTS analysis, this objective function is replaced as follows: For a fixed value of $\beta$, let $r_{(j)}(\beta)$ denote the set of ordered absolute values of the residuals. It is sensible to identify the outliers by using Equation 1 and 2. LTS is obtained from Equation 5 by removing the outliers.

\section{Measures of Accuracy}

Consider the observations $y_{1}, y_{2}, \ldots y_{n}$ and the corresponding estimates $\hat{y}_{1}, \hat{y}_{2}, \ldots \hat{y}_{n}$ generated from a prediction model. The errors are:

$e=y_{i}-\hat{y}_{i}$ for $i=1,2, \ldots n$

Thus, large deviations from the estimates will give large error values. Accuracy measures are defined using these errors. Hence, large measure values imply less accuracy of the prediction model. The measures are the Cumulative Forecast Error (CFE), Mean Absolute Deviation (MAD), Mean Squared Error (MSE), Root Mean Squared (RMSE), standard error $\left(s_{e}\right)$, Mean Absolute Percentage Error (MAPE) (Elamir, 2012) defined as:

$C F E=\sum_{i=1}^{n} e_{i}$ 
This measure adds the errors together and can be used to show if the model is over forecasting above the actual values $(\mathrm{CFE}<0)$ or under forecasts $(\mathrm{CFE}>0)$ :

$$
M A D=\frac{1}{n} \sum_{i=1}^{n}\left|e_{t}\right|
$$

The MAD measure is an average of the absolute errors. Smaller values of MAD show more accuracy of the predictor model while larger ones imply less accuracy:

$$
M S E=\frac{1}{n} \sum_{i=1}^{n} e_{t}^{2}
$$

The MSE measure is an average of the squared errors and has useful mathematical properties related to the variance. Also, smaller MSE values show more accuracy of the predictor model:

$$
R M S E=\sqrt{M S E}
$$

The RMSE measure is the square root of the MSE and the two measures are interpreted in the same way:

$$
S_{e}=\sqrt{\frac{1}{n-1} \sum_{i=1}^{n}\left(e_{i}-\bar{e}\right)^{2}}
$$

The $s_{e}$ measure is a standard deviation of the errors. Smaller values are also indicators of more model accuracy:

$$
M A P E=\frac{100}{n} \sum_{i=1}^{n} \frac{\left|e_{i}\right|}{A_{i}}
$$

The MAPE measure is a percentage of the absolute errors, with smaller values also showing more predictor model accuracy.

\section{Statistical Inference}

Statistical inference refers to the process based on analysed data to deduce properties of an underlying distribution (Held and Bové, 2013). It makes propositions about a population using data. Problems in statistical inference are often related to statistical modeling. Basically, statistical inference conclusions are statistical propositions such as point estimate, interval estimate, rejection of a hypothesis and clustering of data points into groups, among others. In this study, estimation is used and the cut-off measure derived is a result of this system.

\section{Methods}

This was a retrospective cohort study on CD4 counts of HIV positive patients from January 2006 to December 2013 attending treatment at the Tshepang Clinic of Dr. George Mukhari Academic Hospital in Gauteng Province,
South Africa. Tshepang Clinic receives patients from clinics in surrounding townships and villages. The clinic aims to curb HIV advancement and improve ART. The HIV patients' records for the stated period were about 350 , which made the study population. The study used all the useful records of the entire population. Thus the sample consisted of the remaining records (318) after eliminating the unsatisfactorily ones.

\section{Results}

\section{CD4 Count Statistics}

Table 1 shows abridged CD4 counts. The bulk of the values range from $0-200$ and a notable amount is seen from 201-400. Few other values appear to be higher than 401. These may be considered candidates to be outliers.

Figure 1 shows the spread in the CD4 counts and the number of patients. The tail of values is shown from 401 upwards.

\section{Initial Outlier Identification}

Equation 1 and 2 provide the lower and upper limits for outlier identification. Let $k=1.5$ to enhance stable confidence intervals and to avoid $\mathrm{L}$ that is too deep in the negatives. Using the crude quartiles from Table 1, the lower and upper limits are:

$$
\begin{aligned}
& L=Q_{1}-k\left(Q_{3}-Q_{1}\right)=50-1.5(150-50)=-100 \\
& U=Q_{3}+k\left(Q_{3}-Q_{1}\right)=150+1.5(150-50)=300
\end{aligned}
$$

There are no small outliers since the $L=-100$ cells $/ \mathrm{mm}^{3}$ is below all the CD count values in Fig. 1 and Table 1. Actually, all CD4 counts $\geq 0$. Since $L=300$, there are $28 \mathrm{CD} 4$ counts identified as (potential) outliers in class intervals above lower limit 300 in Table 1.

\section{Statistics Grouping for ART Purpose}

Table 2 indicates 247 (77.7\%) bulk of patients below 200 cells $/ \mathrm{mm}^{3}$, who should take ARVs. The next larger group were between 200 and 350 cells $/ \mathrm{mm}^{3}$, which were also recommended to take ARVs. Outliers in this case are only 14 , being the healthy ones $\left(>500\right.$ cells $\left./ \mathrm{mm}^{3}\right)$ and the less severe one sat $350-500$ cells $/ \mathrm{mm}^{3}$.

Table 3 displays the descriptive statistics of the original CD4 count values with their potential outliers.

Table 4 displays the original mean deviation measures of the CD4 counts.

Table 5 lists the outliers identified in the box plot in Fig. 2.

\section{Identifying Outliers}

Robust methods are used as there was evidence of outliers. The analysis identified 18 outliers which were CD4 counts above 300 cells $/ \mathrm{mm}^{3}$. The box plot Fig. 2 identifies them. 


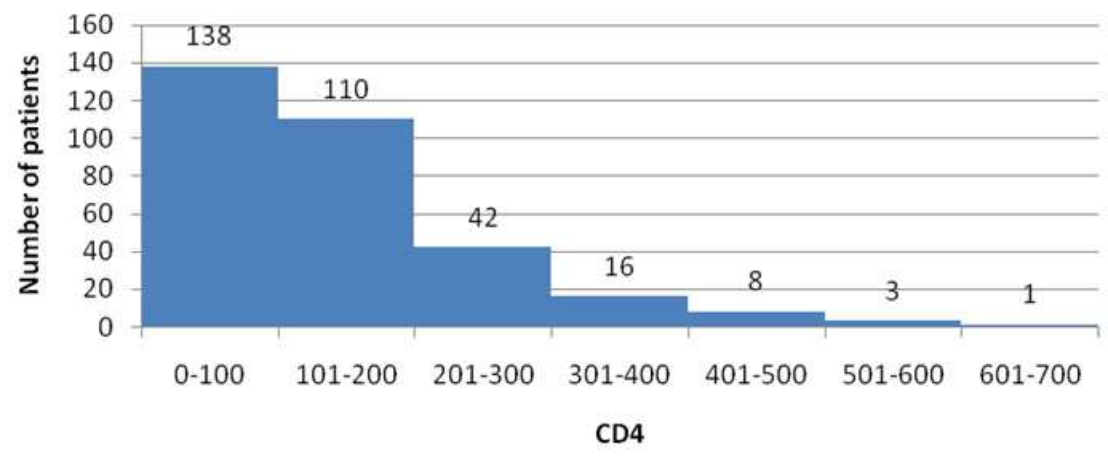

Fig. 1. Shows the spread in the CD4 counts and the number of patients. The tail of values is shown from 401 upwards

Table 1. CD4 counts

\begin{tabular}{lcc}
\hline CD4 & Frequency & Percent \\
\hline $0-100$ & 138 & 43.4 \\
$101-200$ & 110 & 34.6 \\
$201-300$ & 42 & 13.2 \\
$301-400$ & 16 & 5.0 \\
$401-500$ & 8 & 2.5 \\
$501-600$ & 3 & 0.9 \\
$601-700$ & 1 & 0.3 \\
Total & 318 & 100.0 \\
\hline & \\
Table 2. Grouped CD4 count statistics & \\
CD4 & & Frequency \\
\hline 0 to 200 & & 247 \\
200 to 350 & 57 \\
350 to 500 & & 10 \\
500 or more & & 4 \\
Total & & 318 \\
\hline
\end{tabular}

Table 3. Descriptive CD4 count statistics

\begin{tabular}{ll}
\hline Mean & 137.927673 \\
Standard error & 6.238083763 \\
Median & 117.5 \\
Mode & 30 \\
Standard deviation & 111.2409687 \\
Sample variance & 12374.55311 \\
Kurtosis & 2.28654435 \\
Skewness & 1.35006011 \\
Range & 619 \\
Minimum & 2 \\
Maximum & 621 \\
Sum & 43861 \\
Count & 318 \\
Confidence level $(95.0 \%)$ & 12.27327796 \\
\hline
\end{tabular}

Table 4. Original mean deviation measures of CD4 counts

\begin{tabular}{lllll}
\hline CME & MAD & MSE & MAPE & SE \\
\hline$-1.4 \mathrm{E}-05$ & 26949.67 & 3922733 & 930.51 & 6.22 \\
\hline
\end{tabular}

Figure 2 displays the Box plot to identify potential CD4 outliers.

The box plot confirms the possibility of outliers. It also confirms that there are no outliers below the lower limit. However, instead of 18 outliers, 12 outliers identified.
Table 5. CD4 outliers

\begin{tabular}{lll}
\hline$\# 227$ & $=$ & 621 \\
$\# 309$ & $=$ & 539 \\
$\# 32$ & $=$ & 547 \\
$\# 268$ & $=$ & 509 \\
$\# 304$ & $=$ & 489 \\
$\# 222$ & $=$ & 479 \\
$\# 226$ & $=$ & 495 \\
$\# 29$ & $=$ & 439 \\
$\# 38$ & $=$ & 460 \\
$\# 303$ & $=$ & 430 \\
$\# 160$ & $=$ & 405 \\
$\# 253$ & $=$ & 408 \\
\hline
\end{tabular}

\section{Summary Statistics}

The next presentation considers new 'resampled' data when outliers have been removed.

Table 6 uses fewer 'responses'. According to Equation 2, there are still CD4 values above 300 cells $/ \mathrm{mm}^{3}$.

Table 7 displays summary statistics without outliers.

Table 8 displays the original mean deviation of CD4 counts.

Table 9 displays the original mean deviation of less CD4 counts.

Table 10 displays the original mean deviation of less CD4 counts and VL outliers.

\section{Robust Statistical Measures}

The robust measures assessed from the above analyses are LAD, M-estimator and LTS.

\section{Analysis of Results}

Twelve (12) out of 14 measures were affected. Outliers therefore affected $85.7 \%(12 / 14)$ of the measures. This indicates the effect of outliers on the descriptive statistics.

\section{Estimating the Robust Measure Values}

Table 12 displays the original mean deviation of CD4 counts less outliers and leverage points. 


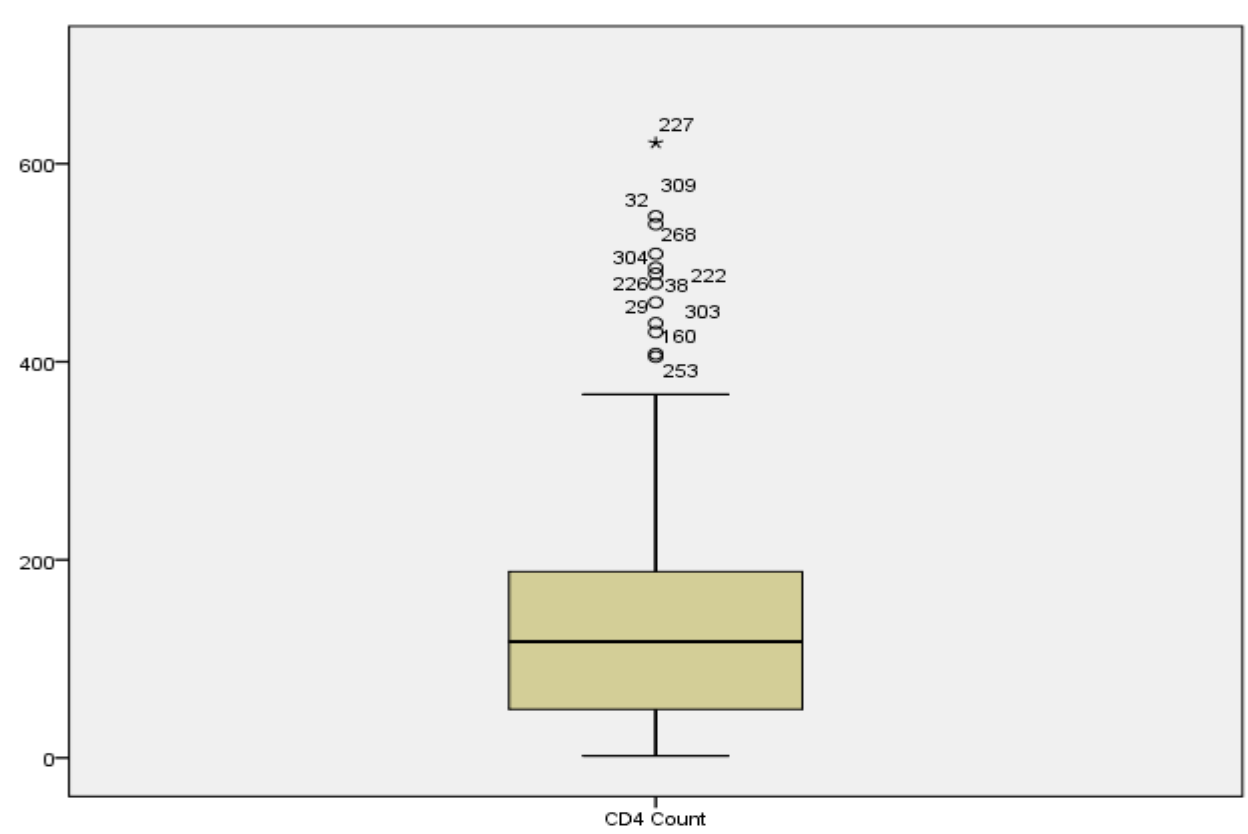

Fig. 2. Box plot of CD4 counts

Table 6. Grouped CD4 counts: Outliers removed

\begin{tabular}{ll}
\hline CD4 & Frequency \\
\hline 0 to 200 & 247 \\
200 to 350 & 57 \\
350 to 500 & 2 \\
Total & 306 \\
\hline & \\
Table 7. Descriptive CD4 counts & \\
\hline CD4 & 124.3137255 \\
\hline Mean & 5.045316852 \\
Standard error & 111 \\
Median & 30 \\
Mode & 88.25699958 \\
Standard deviation & 7789.297975 \\
Sample variance & -0.337132788 \\
Kurtosis & 0.64131174 \\
Skewness & 365 \\
Range & 2 \\
Minimum & 367 \\
Maximum & 38040 \\
Sum & 306 \\
Count & 9.928035022 \\
Confidence level (95.0\%)
\end{tabular}

Table 8. Original mean deviation measures of CD4 counts

\begin{tabular}{lllll}
\hline CME & MAD & MSE & MAPE & SE \\
\hline$-1.4 \mathrm{E}-05$ & 26949.67 & 3922733 & 930.51 & 111.24 \\
\hline
\end{tabular}

Table 9.Mean deviation measures of CD4 counts less CD4 outliers

\begin{tabular}{lllll}
\hline CME & MAD & MSE & MAPE & SE \\
\hline$-3 E-06$ & 22216.15686 & 2375735.88 & 826.9120 & 5.0453 \\
\hline
\end{tabular}

Table 10. Mean deviation measures of CD4 counts less CD4 and VL outliers

\begin{tabular}{lllll}
\hline CME & MAD & MSE & MAPE & SE \\
\hline-0.008 & 21735.8 & 2380897 & 821.8535 & 5.2217 \\
\hline
\end{tabular}

Table 11. CD4 descriptive statistics of different condition

\begin{tabular}{lll}
\hline & Original & No outliers \\
\hline Mean & 137.9277 & 129.8738 \\
Std Error & 6.238084 & 5.550239 \\
Median & 117.5 & 117 \\
Mode & 30 & 30 \\
Std Dev & 111.241 & 96.29305 \\
Sample Var & 12374.55 & 9272.351 \\
Kurtosis & 2.286544 & 1.042803 \\
Skewness & 1.35006 & 0.963119 \\
Range & 619 & 545 \\
Minimum & 2 & 2 \\
Maximum & 621 & 547 \\
Sum & 43861 & 39092 \\
Count & 318 & 301 \\
$95 \%$ c. lev & 12.27328 & 10.92233 \\
\hline
\end{tabular}

Table 12. Mean deviation measures of CD4 counts less outliers and leverage points

\begin{tabular}{lllll}
\hline & MAD & MSE & MAPE & SE \\
\hline Data & 26949.7 & 3922733.0 & 930.5 & 6.2381 \\
No outliers & 22216.2 & 2375735.9 & 826.9 & 5.0453 \\
\hline
\end{tabular}

\section{Discussion}

\section{Least Absolute Deviations}

LAD is the lowest sum of the absolute residual values. From Equation 3, removing outliers leads to the lowest MAD in Table 12. Thus, LAD = 22216.2.

\section{M-Estimators}

M-estimators are estimators obtained as the minima of sums of functions of the data, obtained using Equation 4. These are equivalents of minimum MAPEs using the 
observed values as the divisors, identified in Table 12 . Hence, is M-est $=816.9$.

\section{Least Trimmed Squares}

LTS is a robust statistical method that fits a function to a data set of data, which curtails the sum of squared residuals in Equation 5, also in Table 12. Then, LTS = 2375735.88. The standard error is smaller for 'No outliers' row in Table 11, which seems to confirm that the idea of removing outliers leads to unaffected statistical results.

\section{Discussion and Conclusion}

The values obtained for robust measures are $\mathrm{LAD}=$ $22216.2 ;$ LTS $=2375735.88$ as well as $\mathrm{M}_{\text {est }}=826.9$. The statistical outliers obtained from robust methods and extreme values that are prescribed in the starting cut-off for starting to use ARVs did not indicate to have any relationship. However, the adjustment based on statistical guidelines is possible and can be a useful adjustment in increasing ARV usage by HIV patients in order to manage HIV. More specifically, the value of 300 cells $/ \mathrm{mm}^{3}$ was obtained for an upper bound.

\section{Recommendations}

The study recommends that:

- Health Authorities should revise the starting level of using ARVs to 300 cells $/ \mathrm{mm}^{3}$

The study also recommends that a study:

- $\quad$ Should be conducted determine the CD4 counts that relate to death stage of a HIV positive patient

- Similar to this one should be undertaken on a bivariate sample of CD4 counts and viral load and compare the results

\section{Acknowledgement}

The authors would like to acknowledge Dr M.M Motshwane for recruiting the first author to postgraduate studies in the Department. They would also like to express their appreciations to the unknown referees for their comments and suggestions which improved the presentation of the paper.

\section{Funding Information}

This research was supported and funded by Sefako Makgatho Health Sciences University.

\section{Author's Contributions}

Maria Mokgadi Lekganyane: Conducted the study, contributed to the study methodology, data analysis and interpretation of results. Wrote the paper, reviewed, revised edited and finalised the manuscript.
Solly Matshonisa Seeletse: Supervised the study, collected and developed the initial manuscript. Designed the research plan and organised the study.

\section{Ethics}

This article is original and contains unpublished material. The authors have read and approved the manuscript and no ethical issues are involved.

\section{References}

Alqallaf, F., S. Van Aelst, V.J. Yohaiand R.H. Zamar, 2009. Propagation of outliers in multivariate data. Ann Stat., 37: 311-331. DOI: 10.1214/07-AOS588

Andersen, R., 2008. Modern Methods for Robust Regression. 1st Edn., SAGE Publications, London, ISBN-10: 1412940729, pp: 107.

Dawson, R., 2011. How significant is a boxplot outlier. J. Stat. Educ., 19: 1-12.

Dovoedo, Y.H., 2011. Contributions to outlier detection methods: Some theory and applications. Ph.D. Thesis, University of Alabama, Tuscaloosa, USA.

Elamir, E.A.H., 2012. On uses of mean absolute deviation: Decomposition, skewness and correlation coefficients. METRON, 70: 145-164.

DOI: $10.1007 / \mathrm{BF} 03321972$

Held, L. and D.S. Bové, 2013. Applied Statistical Inference: Likelihood and Bayes. 1st Edn., Springer Science and Business Media, Berlin, ISBN-10: 3642378870, pp: 376.

Li, L.M., 2005. An algorithm for computing exact least-trimmed squares estimate of simple linear regression with constraints. Comput. Stat. Data Anal., 48: 717-734.

DOI: $10.1016 /$ j.csda.2004.04.003

Lichterfeld, M. and E.S. Rosenberg, 2011.Antiretroviral combination therapy markedly reduces risk of heterosexual HIV-1 transmission. Evidence-Based Med., 17: 95-96. PMID: 22108078

Maronna, R.A., R.D. Martin and V.J. Yohai, 2006. Robust Statistics: Theory and Methods. 1st Edn., Wiley, Chichester, ISBN-10: 0470010924, pp: 436.

Sieleunou, I. and M. Souleymanou, 2009. Determinants of survival in AIDS patients on antiretroviral therapy in a rural centre in the Far-North Province, Cameroon. Tropical Med. Int. Health, 14: 36-43. DOI: $10.1111 /$ j.1365-3156.2008.02183.x

Strutz, T., 2010. Data Fitting and Uncertainty: A Practical Introduction to Weighted Least Squares and Beyond. 1st Edn., Vieweg + Teubner Verlag, Wiesbaden, ISBN-10: 3834810223, pp: 244.

Tofallis, C., 2008. Least squares percentage regression. J. Modern Applied Stat. Meth., 7: 526-534. 
Van de Geer, S.A., 2000. Empirical processes in Mestimation: Applications of empirical process theory. Cambridge University Press.
Wilcox, C.W., 2011. Bias: The Unconscious Deceiver. 1 st Edn., Xlibris Corporation,

ISBN-10: 1465342621, pp: 280. 